\title{
Placebo control in Covid-19 trials: A missed opportunity for international guidance
}

URBAN WIESING, HANS-JÖRG EHNI

\begin{abstract}
Vaccines preventing Covid-19 have been approved in several countries. Is it still ethically acceptable to use placebo controls during the development of other vaccine options? If two of the most influential international guidelines of biomedical research are consulted, the Declaration of Helsinki and the CIOMSguidelines, the answer is "no." We discuss the implications for ongoing vaccine research, and how placebo controls might be justified nevertheless. However, the ethical conflict remains highly problematic. We suggest that such ethical dilemmas should be avoided in the future by the introduction of a new system of global governance. Once vaccines are approved, a global regulation should oblige producers to provide the necessary amount of vaccine doses for the control groups of ongoing vaccine research.
\end{abstract}

Key words: Research ethics, Declaration of Helsinki, CIOMS Guidelines, placebo control, Covid-19 vaccine

\section{Introduction}

From time to time an interesting phenomenon can be observed in medical ethics: Ethicists struggle with a problem for a long time without finding a solution acceptable to all. Then the problem may disappear for a certain time, only to suddenly resurface at the centre of a current debate. One such problem is the ethical permissibility of placebo controls in clinical trials even if a standard therapy is available. This question is gaining urgency yet again in Covid-19 vaccine trials, as recently approved vaccines have become available.

Authors: Urban Wiesing (corresponding author - urban.wiesing@unituebingen.de), Director, Institut für Ethik und Geschichte der Medizin, Gartenstrasse, Tübingen, GERMANY; Hans-Jörg Ehni (hans-joerg.ehni@unituebingen.de), Deputy Director, Institut für Ethik und Geschichte der Medizin, Gartenstrasse, Tübingen, GERMANY.

To cite: Wiesing U, Ehni H-G. Placebo control in Covid-19 trials: A missed opportunity for international guidance. Indian J Med Ethics. 2021 Apr-Jun; 6(2) NS: 118-121. DOI: 10.20529/IJME.2021.22.

Published online first on March 22, 2021.

Manuscript Editors: Ruth Macklin, Amar Jesani.

Peer reviewer: An anonymous peer reviewer.

(c) Indian Journal of Medical Ethics 2021
In the literature, this problem has been discussed extensively in the context of studies in sub-Saharan Africa and Thailand (1). There, the prevention of HIV transmission from mother to unborn child was tested against placebos, although a costly and complicated standard therapy was available in wealthy countries. This therapy, however, was not available in poor regions of the world. Proponents of placebo controls argued that, in such cases, these were methodologically superior and that clinical researchers had no obligation to offer a therapeutic option in research. Moreover, they argued, in poor regions the risk-benefit ratio of participation in the trial would be no worse than that of non-participation. However, their opponents considered it an obligation to offer existing therapeutic options as comparator, feared the exploitation of poor countries for the benefit of the rich, and set a very low threshold for acceptable risks in this context. As is often the case in applied ethics, neither side could fully prevail.

The issue led to several revisions of the Declaration of Helsinki (DoH). After lengthy discussions, a compromise was reached in Fortaleza for the 2013 version, which a majority of delegates to the General Assembly of the World Medical Association accepted (2). The CIOMS guidelines were also adapted accordingly, to say: If a standard therapy is available, trials with a placebo may proceed only for compelling scientific reasons and with low risks involved (3). The actual controversy subsided without being resolved decisively.

Now the question of whether placebo controls are ethically permissible has arisen again within the context of Phase 3 trials of vaccines against Covid-19. The development of various vaccines has been dramatically fast, in that Phase 3 trials were ongoing when the medical approval of one vaccine in December 2020 and another in January 2021 changed the situation. The question arises equally for trials starting after the first approval of a vaccine: Should ongoing placebo-controlled trials be allowed to continue unchanged? And should additional placebo-controlled trials be permitted? What do the Declaration of Helsinki (DoH) and CIOMS guidelines say about this?

The authors are aware that both documents have a different status in different countries. The spectrum ranges from vague recommendations, soft law to legislation. Therefore, one cannot readily conclude that there is a legal obligation in a 
particular country to follow these documents. However, the ethical principles underlying the two documents are worth discussing as global principles, whatever their legal status may be, as they are among the most influential international guidelines for biomedical research.

\section{Newly licensed vaccines as a required active control?}

The compromise as formulated in the guidelines was first to define the comparator to be used as a rule. $\$ 33$ of the Declaration requires the "best proven treatment(s)", CIOMS Guideline 5 demands an "established effective intervention" (3: p 15).

Unless the new vaccines fall within the category of comparator defined above, placebo controls would be legitimate. One may test against a placebo, according to the $\mathrm{DoH}$, "where no proven treatment exists". The CIOMS guideline requires an "established effective intervention" as a comparator and thus defines the requirements somewhat more broadly. This includes not only the "best proven treatments" but also possible alternatives that may be somewhat inferior; but which a physician could reasonably prescribe on the basis of existing evidence.

At least in the case of emergency approval for an intervention, it could be argued that this could not yet be considered to set a standard and its experimental character would remain. However, even in the case of emergency approval of the first Covid-19 vaccine, it constitutes an intervention for which there is no other alternative, and thus the only treatment option for which there is at least some evidence. However, with a regular approval, a new standard is set where no treatment option existed previously. How long that standard has been in place, or how widespread access to it is does not change the fact that it is a standard, considering the scientific evidence. Now multiple vaccines have been approved for Covid-19, and not just via emergency approval. This means that there is at least one comparator that meets the conditions of both the DoH and the CIOMS guidelines.

\section{Necessary conditions for exceptions}

According to the $\mathrm{DoH}$ and the CIOMS Guidelines, if a comparator is available, testing against a placebo in a study may only be performed if two conditions are met:

(i) The risk posed by using a placebo instead of a comparator must not be greater than a "risk of serious and irreversible harm" according to the DoH. The CIOMS guidelines set the limit at a "minor increase over minimal risk".

(ii) There must be compelling scientific reasons $(\mathrm{DoH}$ : "compelling and scientifically sound methodological reasons", CIOMS:"compelling scientific reasons").

Are these conditions fulfilled?

Already the first condition is not fulfilled. Contracting Covid-19, especially in the case of older participants in the study, is a higher risk than either guideline allows. This is because it is potentially lethal. Even a milder course of the disease is associated with irreversible long-term damage, such as the loss of the sense of smell or taste and reduced physical performance. Since both conditions must be fulfilled, there would already be sufficient reason to reject placebo controls in the present case.

The second condition is also not met. The efficacy of a new vaccine could be determined by testing against an existing vaccine. Only, it would be more laborious and would probably produce clear results less quickly. However, these are not "compelling scientific reasons" in the sense that compelling means necessary or indispensable.

Thus, the two conditions CIOMS and DoH specify for exemptions are not met. Accordingly, any further Phase 3 trials of Covid-19 vaccine ought not to be tested against a placebo after the first approval of a vaccine. Strictly speaking, ongoing trials would also have to be modified in their design.

\section{Exceptions to the rule?}

However, these guidelines are countered by reality: Approved vaccines are in very short supply. Moreover, the world is currently in an exceptional situation. The current pandemic is characterised by the global emergency caused by a highly contagious disease which has led and continues to lead to a high burden of deaths and economic damage. The pandemic is having the most profound impact on people's lives in decades. So far it has led to more than two million deaths globally, and can only be contained by contact restrictions and quarantine. Moreover, there is currently no effective treatment for Covid-19 infections. This implies that the social value of new therapeutic and preventive interventions is exceptionally high. Therefore, one question comes to mind: The DoH and CIOMS guidelines are necessarily written in general terms. Are they sufficiently differentiated to be appropriate in this exceptional situation? And the general question remains: Are there sufficient ethical reasons to deviate from the guidelines? Corresponding arguments for further placebo-controlled Phase 3 trials are mentioned by a WHO Ad Hoc Expert Group on the Next Steps for Covid-19 Vaccine Evaluation (hereinafter WHO Ad Hoc Expert Group) (4), and by Wendler et al (5).

One could argue that both sets of rules specify an absolute upper limit of the risk that may be taken only regarding placebo control. For research without a placebo they do not specify an upper limit of acceptable risk. If necessary, they weigh the risk for study participants without possible benefit against the benefit for future patients and society. In the current situation, one could argue that the benefit to society from another vaccine is exceptionally huge. Moreover, a pandemic in which an approved vaccine is not even widely available in most countries, and alternative vaccines are urgently needed, constitutes a special emergency.

Assuming that the placebo paragraphs of the $\mathrm{DoH}$ and the CIOMS guideline are inappropriate in the current exceptional 
situation, one could try in turn to keep the risk to the participants in the studies as low as possible. This would not resolve the issue, but it would reduce the potential conflict. Which options could lower the risk to participants in a placebo-controlled trial? Within post-study arrangements, trial participants in the placebo group could be offered vaccination immediately after the study, which would reduce their overall risk of contracting Covid-19. Minimising the amount of time for which a participant receives the placebo only and assigning only those participants to the placebo group who are not at increased risk for severe Covid-19 might reduce the risk of the study. Vaccine trials could include only healthy volunteers. Based on the argument of consistency, the acceptable risk for them may be set as high as is usually done in Phase 1 trials. However, both a premature discontinuation of the placebo group and a selection of particularly healthy participants could reduce the explanatory power of a study or bias the results.

In addition, further studies could take longer, so that there might be a ready inclination to lessen the requirements for approval. However, one should be warned against this. A "pandemic research exceptionalism", as described by London and Kimmelmann (6), a renunciation of "rigorous methods" in the study design and therefore the reduced possibility to obtain "unbiased effect estimates" would not be justifiable ethically. Many more people would be exposed to the risk of a poorly tested vaccine compared to those who face the risk of a placebo control in a trial. It would also be detrimental if studies with low explanatory power undermined the confidence in research and in approved vaccines.

Notwithstanding measures that reduce the risk to participants in the placebo group, they would also need to be informed about the placebo control. For ongoing trials, the participants would need to be informed about the new circumstances, given the option of stopping, unblinding, continuing, and of special post-study arrangements. Participants in the placebo group should receive a vaccine after the study that has been successfully tested as effective.

\section{What does this mean for different studies?}

1) What should happen if a competitor's vaccine is approved during an ongoing trial? Provided that a particular study is still conducted in the phase of scarcity of the other approved vaccine, a continued placebo control could possibly be justified by the fact that an active control is not possible and another alternative vaccine would be required urgently especially with regard to the said scarcity, the progression of the pandemic, and the efficacy of different vaccines with different virus variants. A placebo control might also be justifiable, particularly for study participants who, as healthy volunteers, do not belong to any risk group and do not belong to any other priority group for vaccination. The period until all participants receive the vaccine ought to be kept as short as possible. However, in line with the above argumentation, care is needed to maintain the scientific validity of such a study. Without doubt, study participants would have to be informed about any recently approved comparator.

Should a vaccine become widely available during or at the beginning of a study, likely far fewer subjects would be willing to possibly receive a placebo instead of an effective vaccine. Many might drop out of an ongoing trial. Only low-risk subjects are likely to participate in a new trial. This, too, could reduce the explanatory power of a study.

2) A second case would be a study that is initiated after the approval of one or more vaccines. As such, a placebo control would be more difficult to justify, especially if it is foreseeable that the vaccine(s) will become widely available during the ongoing study. The most important argument that could be made for a placebo control is the faster approval of a further vaccine, that is, a benefit to society. In principle, this also applies to the particular case when the vaccine is already approved and widely available in one country, while a trial is just being initiated in another country to explore a local alternative.

\section{The international dimension}

Neither the Declaration of Helsinki nor the CIOMS guidelines provide for an exception for placebo controls due to lack of local availability. However, this is precisely what the WHO Ad Hoc Expert Group found acceptable: "Countries with limited or no access to a known effective vaccine could thus ethically permit placebo-controlled trials of vaccines of potential relevance to them even if effective vaccines were already being marketed elsewhere." (4). Permission to pursue different placebo regimes because of regional differences, most of which are also associated with different levels of wealth, is likely to face vigorous political opposition from poorer countries and from advocacy groups, as with the HIV trials mentioned at the beginning.

In summary, one can certainly find arguments opposed to continuing placebo controls when a comparator is present, as also arguments in favour. However, one must be aware that in such cases one deviates from the requirements of the DoH and the CIOMS guidelines. Admittedly, this involves an important disadvantage. Both documents represent a hardwon political consensus that should not be abandoned lightly. The specific rules for placebo controls came about as a result of significant pressure from poorer countries. They saw an increased risk of abuse if placebo control was allowed there, while studies in wealthy countries would test against the standard therapy. Moreover, patients in rich countries might stand to benefit the most from the results of such studies. Poor countries feared exploitation for the benefit of rich countries. This led to the rules in the DoH and the CIOMS Guidelines. The international validity and standing of the documents would be eroded if they were deviated from in the current situation with potentially detrimental consequences for common trust in the DoH and the CIOMS Guidelines.

At the same time, what was stated at the beginning remains true: Proponents and opponents of placebo control despite 
the existence of a comparator will likely not come to an agreement, not even in this pandemic. Both sides can put forward viable arguments for their position.

\section{Global injustice}

Ultimately, the question of placebo control points to the basic problem of all medical research on human participants: How much risk to participants can be accepted to offset a gain in knowledge? If there is continued disagreement about a problem, the better route is not to let the problem arise at all. And placebo controls due to the scarcity of approved, i.e. licensed, vaccines could have been avoided. A sensible governance of Covid-19 vaccine research would have prevented additional placebo-control risks in the first place. The debate results from a fundamental problem with global research and global healthcare: The WHO has complained that the vaccine was not distributed as part of a globally consolidated effort, at the expense of poor countries, and that there was no plan to vaccinate against the pandemic in a coordinated way around the world. In our opinion, this is a political and moral scandal.

This criticism also applies to the coordination of research, and thus to the problem of placebo control: the crucial precondition of the conflict is the limited availability of the standard therapy. So, what percentage of currently available doses would need to be allocated to research in ongoing and upcoming Phase 3 trials to avoid the problem of placebo control? Certainly, only a small percentage. Not making the vaccine available for research is an allocative failure. With several hundred million doses, it should be possible to make a few 100,000 available for research as a comparator. This number would be in the per mille range of the total. And those participants who would receive the standard therapy as a comparator would also contribute to increasing the vaccination rate in a society. For all the methodological advantages that a placebo-controlled trial may have, the decisive factor here is whether a vaccine compares in effectiveness to an already approved alternative and not merely to a placebo.

\section{Globalised research lacks adequate regulatory competence}

The problem was foreseeable. It could have been stipulated at the time of the first approval that a certain, small percentage of a vaccine must be made available for further research as a comparator. But there is no globally operating institution that could decide and implement this.

In this respect, we are dealing with a lack of regulatory competence at the global level. It is a collective failure that no authorised and empowered global institution for global problems exists. One could have solved the problem simply by making a per mille portion of vaccines available immediately for further research. But this did not happen, as no agency is responsible. The placebo problem with Covid-19 studies clearly shows that for global research there is no institution that can take responsibility at a global level. If any lesson can be taken from this issue, it is this: Create institutions that have adequate powers and can respond effectively to research structures! A global pandemic urgently requires the effective global regulation of research.

\section{References}

1. Ehni H-J, Wiesing U. International ethical regulations on placebo-use in clinical trials: a comparative analysis. Bioethics, 2008 Jan; 22(1), 64-74. Doi: 10.1111/j.1467-8519.2007.00587.x

2. World Medical Association, WMA Declaration of Helsinki-Ethical Principles for Medical Research Involving Human Subjects. 2013 Oct [cited 2021 Mar 2]. Available from: https://www.wma.net/policies-post/ wma-declaration-of-helsinki-ethical-principles-for-medical-researchinvolving-human-subjects/

3. Council for International Organisations of Medical Sciences (CIOMS) and World Health Organization (WHO). International Ethical Guidelines for Health-related Research Involving Humans. Geneva: CIOMS and WHO; 2016[cited 2021 Mar 2]. Available from: https://cioms.ch/wpcontent/uploads/2017/01/WEB-CIOMS-EthicalGuidelines.pdf

4. WHO Ad Hoc Expert Group on the Next Steps for Covid-19 Vaccine Evaluation, Placebo-Controlled Trials of Covid-19 Vaccines - Why We Still Need Them. N Engl J Med. 2021 Jan 14; 384(2): e2-3. Epub 2020 Dec 2.

5. Wendler D, Ochoa J, Millum J, Grady C, Taylor HA. COVID-19 vaccine trial ethics once we have efficacious vaccines. Science. 2020 Dec 11; 370(6533):1277-9.

6. London A J, Kimmelman J. Against pandemic research exceptionalism. Science. 2020 May 1;368 (6490): 476-7. Doi: 10.1126/science.abc1731. Epub 2020 Apr 23.

\section{Be a part of IJME}

IJME invites readers to submit research studies, comments, case studies, reports, reviews, letters, as also poems, short stories, original paintings and photographs of print quality ( both in colour and B/W ) to be considered for publication.

All submitted matter is subject to peer review.

Contributors are neither paid nor charged any fee for published matter. 